Kalpa Publications in Computing
Volume 13, 2019, Pages 86-95
Proceedings of the 1st International Con-
ference on Geospatial Information Sciences

\title{
Protocol for the Photogrammetric Survey of Buildings Using Unmanned Aerial Vehicles
}

\author{
José Roberto Hernández Castro \\ CentroGeo Campus CDMX, CDMX, México. \\ jhernandez@centrogeo.edu.mx
}

\begin{abstract}
Photogrammetry encompasses imagery interpretative and measurement method to obtain the shape and location of an object. Since the beginning of the Digital Photogrammetry era (late 80 's), the three-dimensional reconstruction of objects has become one of its fundamental goals [Luhmann et al., 2014]. In the last few years, the professional use of Unmanned Aerial Vehicles (UAVs, also known as drones) focused on this goal has increased and matured relatively fast.

The purpose of this work was to know if and how the UAVs have changed Photogrammetry: how they have modified the Photogrammetric Process and what the necessary bases are to establish a Protocol that provides the necessary steps to make high quality Photogrammetric Surveys of build-ings.

Thanks to the CentroGeo-INEGI project "Desarrollo y Evaluación de Técnicas Avanzadas de Percepción Remota para Alimentar un Catastro Tridimensional", a team and the author performed a Photogrammetric Survey (of the Ajusco Unit of the National Pedagogical University [UPN] in Mexico City) to evaluate the Protocol.

The author prepared this work as a thesis to obtain the degree of Geomatics Engineer from the National Autonomous University of Mexico (UNAM). For more information, check the TesiUNAM website: shorturl.at/fkBQX
\end{abstract}

\section{Introduction}

Photogrammetry can be classified, according to the imaging system used, in Aerial and Terrestrial Photogrammetry. In high-density urban cities, Terrestrial Photogrammetry is limited only to building facades and becomes useless for the identification of land limits [Jazayeri et al., 2014].

Photogrammetry is use in many areas such as Cartography, geophysical and geo-logical studies, urban planning, cadastral studies, design and construction of highways and natural resources assessments [Caire Lomelí, 1977]. The technological development and the evolution of Photogrammetry have promoted the increase of study areas in which Photogrammetry can be apply.

O. S. Siordia, J.L. Silván Cárdenas, A. Molina-Villegas, G. Hernandez, P. Lopez-Ramirez, R. Tapia-McClung, K. González Zuccolotto and M. Chirinos Colunga (eds.), iGISc 2019 (Kalpa Publications in Computing, vol. 13), pp. 86-95 
Globalization has promoted the increased need to obtain and share a large amount of information in short times. Unmanned Aerial Vehicles (UAVs also known as drones) have emerged as a tool that allows that. For its functionality, speed of processing and innovation in the products that can be obtained, they possess a broad field of applications in the urban environment.

The removal of a third person, the pilot of the aircraft, has allowed a change in the way that a photogrammetric flight can be planned. The increase on the research and the development of specialized software have produced an increment of the UAVs' professional applications. Those applications now go from the creation orthomosaics that work as base maps, to high-resolution 3D models generation.

\section{Photogrammetric Process}

The Photogrammetric Process includes all the steps that have be followed to perform a Photogram-metric Survey. Both the Process and the steps have evolved hand in hand with the eras of Photo-grammetry. In the current era (Digital Photogrammetry), the Photogrammetric Process is summarize with the diagram of Figure 1.

The beginning of the Photogrammetric Process is the election of the place (or object) of study. After this decision has been made, the First Step consist of carrying out all the previous work of Flight Planning, for the sub-sequent election of the type of system with which the information will be ob-tained (Second Step) and the software that will be used for its processing (Third Step) and obtaining results (Fourth Step). The Photogrammetric Process ends by making an evaluation of the accuracy and quality of the obtained Photogrammetric Products.

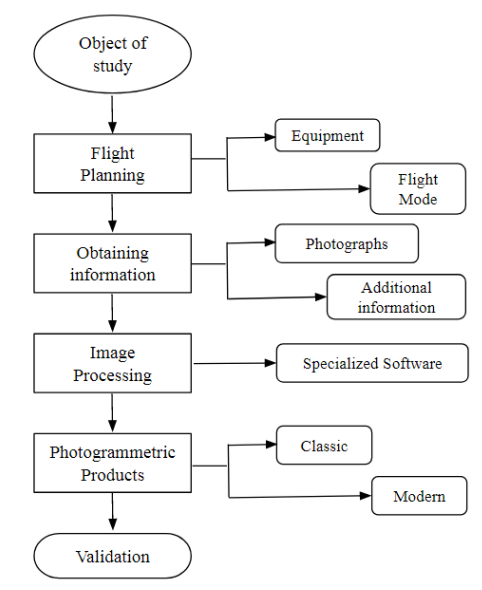

Figure 1: Digital Photogrammetric Process

\subsection{Flight Planning}

For the correct execution of a Photogrammetric Survey, it is necessary to prepare a thorough planning prior to performing any work. The first (and probably most important) decision to make is to decide which products you want to obtain in the Process, as well as their scales and their level of precision [Wolf et al., 2014]. In the specific case of Aerial Photogrammetry, the planning can be summarized in three stages:

1. Photogrammetric Flight Planning, 
2. Land Control Planning, and

3. Selection of the equipment and processes necessary to obtain the desired products. Step.

The Flight Planning step lays the groundwork for everything that has to be done in the Second

\subsection{Obtaining Information}

In the second step, reliable information about the properties of the surface and the objects in it, through processes of recording electromagnetic energy patterns, predominantly in the form of photographs [Schenck, 2005]. Besides the photographs, additional information must be established in this step. This information consists of Ground Control Points (GCPs) that will be use to give geographic reference to the photographs, or to adjust this information if Inertial Navigation (GPS/INS) data is available, like in this work. The need for additional information depends entirely on the main objective of the Survey and Photogrammetric Products.

\subsection{Image Processing}

Once the photographs and the necessary information are obtained, all of the data must be processed using specialized photogrammetric software. Currently, there are many programs suitable for the Digital Photogrammetric Process.

\subsection{Photogrammetric Products}

The Photogrammetric Products are obtained in the last step of the Photogrammetric Process. They have been increasing and evolving with the development of digital technologies, nowadays Digital Photogrammetric Products have advanced to such a degree that they can compete with those generated by means of laser scanning, which have a higher cost and require a greater specialization for its correct use [Remondino \& El-Hakim, 2006].

As a proposal for the categorization of products, the classification in Classic Photogrammetric Products and Modern Photogrammetric Products was presented. Although both classes can be obtained with specialized software, the information that could be obtained in Traditional Photogrammetry is highlighted in the first class. The two key products found within the Classic category are orthophotographs and orthomosaics. In the case of the Modern Products, all those that can only be obtained with the latest generation software are grouped in:

1. 3D Point Clouds

2. Three-dimensional model; that can be classified in:

o Digital Surface Models (DSM),

o $\quad$ Digital Terrain Models (DTM), and

o 3D models of buildings or constructions.

\section{Protocol}

Protocol is defined in the Merriam-Webster Dictionary as a detailed plan of a scientific experiment or procedure. For this work, the proposed Protocol consisted of a breakdown of all the activities and processes necessary to obtain high quality and reliable Photogrammetric Products from buildings, generated by using UAVs. 
Applying as a sequential basis the Digital Photogrammetric Process Diagram (Figure 1), the Proto-col for the Photogrammetric Survey of Buildings using Unmanned Aerial Vehicles has the struc-ture shown in Table1.

\begin{tabular}{|c|c|c|c|c|c|c|}
\hline Building & Equipment & Flight Mode & Survey & Processing & Products & \\
\cline { 1 - 4 } $\begin{array}{c}\text { Geometry and } \\
\text { Accessibility }\end{array}$ & UAV & Free & Flight & & Classic & Validation \\
\hline $\begin{array}{c}\text { Legal } \\
\text { Regulations }\end{array}$ & Additional & Programmed & GCPs & Software & Modern & \\
\hline
\end{tabular}

Table 2: Protocol for the Photogrammetric Survey of Buildings using Unmanned Aerial Vehicles.

The protocol is classified in seven categories. The first one is focused on the building of study, its geometry and near vegetation, and all the legal documents needed to do a professional Photogrammetric Survey. The second category is mainly focused on the required equipment, the UAV and additional (like GPS antennas). The third one refers to all the fieldwork (UAV flights and Ground Control Points establishment). The fourth category refers to the processing of all the information acquired in the survey, done primarily with specialized software. The last two categories refer to the obtained products and their validation.

To do an evaluation of the proposed Protocol, it had to be applied in a Photogrammetric Survey. Thanks to the CentroGeo-INEGI project Development and Evaluation of Advanced Remote Sensing Techniques to Feed a Three-dimensional Cadaster, access to the facilities of the National Pedagogical University (UPN) Ajusco Unitwas obtained.

In the following sections, the protocol categories are further described and their application is shown in the case of the UPN.

\subsection{Geometry and Accessibility}

For this work, it was proposed that a building could be classified by its Geometry as Regular or Com-plex. The buildings of the UPN Ajusco Unit have a complex structure, since they were built using chiseled concrete with marble grain and designed with a geometry that makes a distant reference to Pre-Columbian Mexico [Reyes Salas, 2013].

Accessibility was considered as the amount of vegetation present around a construction. This aspect was of great importance since the dense vegetation near buildings causes shadows, which decreases the amount of information that can be obtained [Calvo, 2015]. The UPN Ajusco has a high vegetation density, fortunately most of it is concentrated in the same area and the facades of the main buildings have a high degree of architectural appreciation.

\subsection{Legal Regulations}

Depending on the country, legal regulations may vary. In the specific case of Mexico, the pilot must have a third-party damage insurance and have the UAV registered with the General Directorate of Civil Aviation.

\subsection{Equipment}

In addition to the access to the University facilities, thanks to the project and Centro-Geo, all the necessary equipment for obtaining images and their processing were available:

1. One UAV (quadcopter) - DJI Inspire 1 
2. One iPad Mini with the Pix4D Capture app

3. Two GPS NAVCOM SF-3040 antennas

4. One tripod and two (2 meters) ranging rods (with bipods)

5. One laptop (Acer Aspire VX) for image processing with Pix4DMapper Pro and MATLAB licenses

\subsection{Flight Mode}

Flight Mode is the way in which a UAV performs a Photogrammetric Survey; they are classified in two groups: Programmed and Free Flight. The first ones being when the pilot has a semiautonomous control of the UAV and the latter being when the pilot has full control of the UAV and the camera. Programmed Flights are recommended for Photogrammetric Surveys.

For this work, the Flight Mode was selected with the Pix4D Capture app for iOS, which has four different types of programmed flight modes: Polygon, Circular, Grid and Double Grid. Based on the results of previous works carried out in the project, it was concluded that due to the large land extension and the size of the main buildings the most convenient Flight Mode was Double Grid.

In addition to the land extension, the battery life (approximately 22 minutes of flight each) had to be considered. It was decided to make more than one flight and merge them all in the processing.

\subsection{Survey}

The Photogrammetric Survey is divided in flight (or flights) and in the establishment of the Ground Control Points.

Flight. For this work, the team and the author made three Double Grid flights with the following characteristics:

1. Flight height: $70 \mathrm{~m}$

2. Flight speed: $6 \mathrm{~m} / \mathrm{sec}$

3. Angle of camera: $70^{\circ}$

4. Front overlap: $80 \%$

5. Side overlap: $70 \%$

6. Look at grid's center: OFF

7. Picture trigger mode: SAFE MODE

8. White balance: $\mathrm{ON}$

\begin{tabular}{|c|c|c|}
\hline Flight & Time of Flight & No. of photos \\
\hline 1 & $13 \min 21 \mathrm{sec}$ & 150 \\
\hline 2 & $15 \min 6 \mathrm{sec}$ & 243 \\
\hline 3 & $15 \min 31 \mathrm{sec}$ & 385 \\
\hline Total & $4 \min 58 \mathrm{sec}$ & 628 \\
\hline
\end{tabular}

Table 3: Time of positioning and antenna height. 
The results of each flight were:

Ground Control Points. The Ground Control for this work was made using two NAVCOM SF3040 antennas. In total there were five GCPs, four of them were pre-marked with artificial targets. These objectives were square canvases of four square meters designed during the tests prior to this work. The specific characteristics of the GPS surveys were:

1. Positioning Method: Static

2. Tracking: GLONASS and GPS

3. Elevation mask: $20^{\circ}$

4. Antenna Type: NAVCOM SF-3040

5. Instrument height: Variable

6. PDOP Limit: 5.0

7. Time interval: 2 seconds

The results of the times of positioning for each GPS were:

\begin{tabular}{|c|c|c|}
\hline GCP & Time of Positioning & Antenna Height \\
\hline GCP 1 & 1 hour & $1.978 \mathrm{~m}$ \\
\hline GCP 2 & 47 minutes & $2 \mathrm{~m}$ \\
\hline GCP 3 & 1 hour 55 min & $1.872 \mathrm{~m}$ \\
\hline GCP 4 & 46 minutes & $1.812 \mathrm{~m}$ \\
\hline GCP 5 & 1 hour 9 min & $1.856 \mathrm{~m}$ \\
\hline
\end{tabular}

Table 3: Time of positioning and antenna height.

The raw data of the coordinates of each point were processed individually in Star- Point, with vectors created with the Queretaro and Toluca stations of the RGNA (Na-tional Active Geodetic Mexican Network). The result of the adjustment of the net-work had a reliability of $95 \%$. The (geographic) corrected coordinates were projected into UTM 14 N.

\subsection{Processing}

The 628 images were uploaded to Pix4DMapper Pro to be processed with the 3D Models template. The GCPs were used to make the spatial resection of the photo-graphs. As mentioned earlier, the processing was done on an Acer Aspire VX laptop with 16GB of RAM and an NVIDIA GeForce GTX 1050 Ti graphics card. This was of utmost importance since Pix4D requires as many resources as possible.

The processing was performed semi-autonomously with the 3D Models template. The only process done "manually" in Pix4D was the correction of the georeference, this was done by selecting the exact pixel were each GCP was visible. The number of images varied from GCP to GCP. Once this step was done, the project was re-optimized. The result of this process was correct, with a Root Mean Square (RMS) error of $5.5 \mathrm{~cm}$ (less than the double the value of the Ground Sample Distance (GSD) of $3.22 \mathrm{~cm}$ ). The GSD is the distance between two consecutive pixel centers. The higher the 
value, the lower the spatial resolution of the image. The GSD is completely related to the flight height: the higher the altitude, the greater the value of the GSD [Calvo, 2015].

Since the georeferencing was correct, the project was re-optimized to update the coordinates of the Point Cloud.

\section{Products}

\subsection{Point Cloud}

After the re-optimization of the project, the first obtained product was the Point Cloud. The 3D Point Cloud of this work consisted of 33,415,947 points. The pro-cessing time to convert the 628 photographs into a dense cloud was 10 hours and 39 minutes. Once the Point Cloud was obtained, it was processed in MATLAB with the UIPCPV user tool (developed by Dr. José Luis Silván Cárdenas in CentroGeo) to clas-sify it. The Point Cloud was categorized in five different classes:

1. Ground,

2. Bare ground,

3. Low vegetation,

4. High vegetation, and

5. Build.

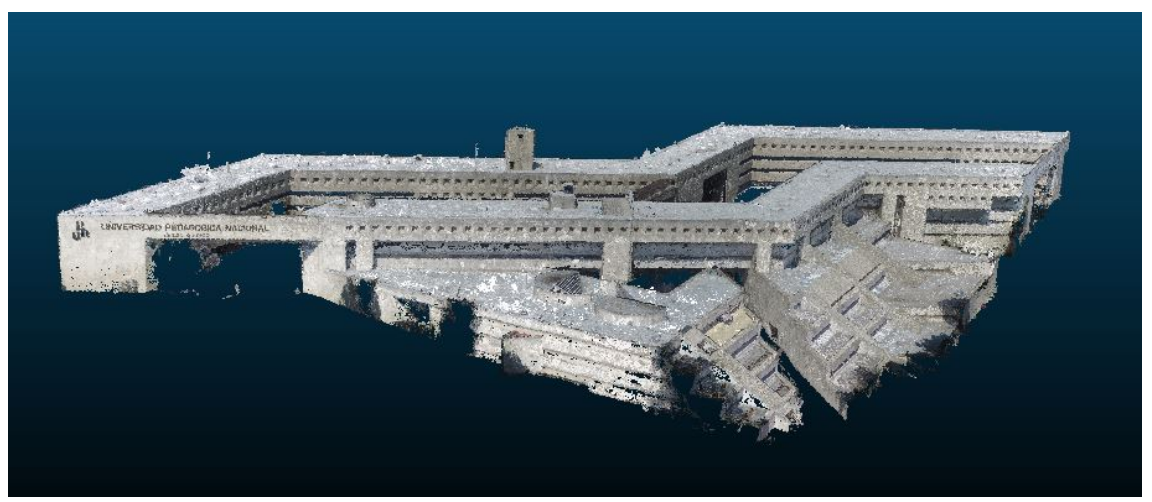

Fig. 3: UPN Ajusco Point Cloud, Build class.

\subsection{D Mesh}

In Pix4DMapper Pro, the 3D Mesh is generated from a triangular mesh that joins each one of the points of the Cloud. For this work, the time of its generation was 30 minutes and 12 seconds.

\subsection{Orthomosaic}

The Orthomosaic is the only classic product that can be obtained through Pix4D. The spatial resolution of the Orthomosaic (and the Digital Surface Model [DSM]) was the GSD, $3.22 \mathrm{~cm}$. The processing time of the Orthomosaic generation was two hours and 20 minutes. The Orthomosaic was the product chosen to evaluate the accuracy of $2 \mathrm{D}$ position. 


\subsection{Digital Surface Model}

The obtained DSM is a great tool to perceive the presence of high dense vegetation in the University Facilities and the behavior of the land. It was generated based on the height information of each of the points in the Cloud. The generation time of the model was 48 minutes and 52 seconds.

\section{Validation}

The georeference of a Photogrammetric Project obtained through GCPs can be evalu-ated to measure the accuracy of the position. The required product to do this valida-tion was the orthomosaic, which (for this process) was handled as a high-resolution image. In order to perform the validation, it is necessary to have Independent Control Points (ICPs). A GPS survey must take place, like in the case of the GCPs, yet their number and distribution must be completely different from the GCPs. Its main objec-tive is to evaluate the planimetric accuracy in sensor orientation and orthorectifica-tion [Cruz Reyes, 2015].

The Federal Geographic Data Committee (FGDC) states that to assess the plani-metric accuracy of a high resolution images, a minimum of 20 ICPs (distributed in the study area) must be established [Lencinas \& Díaz, 2011]. The Planimetric Accu-racy in this work was validated by calculating the RMS of the differences in the posi-tion of each ICP in the Orthomosaic. The ICPs survey was done with the same equipment as the GCPs (NAVCOM SF-3040) but with a different positioning method. The information of the five GCPs were used as the basis for a kinematic survey. In total, 29 ICPs were surveyed.

To calculate the RMS of the position, the surveyed ICPs coordinates were com-pared with the coordinates of same place in the orthomosaic. At the time of making this com-parison, it was observed that one of the ICPs was outside the orthomosaic area, so it was eliminated. There were complications with five more ICPs, due to the value of the GSD and the flight height its correct identification in the orthomosaic became complicated. Therefore, the calculation of the RMS was performed with 23 ICPs. The calculation was performed with the use of a digital spreadsheet. The lati-tude and longitude differences were calculated separately. Subtractions of the or-thomosaic coordinates mi-nus the actual coordinates were performed, these differ-ences were squared; the sum of all the differences was made; the number of ICPs divided this amount. At this point, the RMS of $\mathrm{x}$ and $y$ were obtained. To calculate the RMS2D, the root of the sum of the square of the horizontal and vertical RMS was calculated.

The result of the RMS2D was $0.2077 \mathrm{~cm}$. To decrease this value, the three ICPS whose residue at $\mathrm{x}$ or at $\mathrm{y}$ was greater were eliminated. This elimination was done in such a way that the FGDC rule of having a minimum of 20 ICPs will continue to be fulfilled. When working with only 20 ICPs, the result of the RMS2D was $0.1555 \mathrm{~cm}$. 


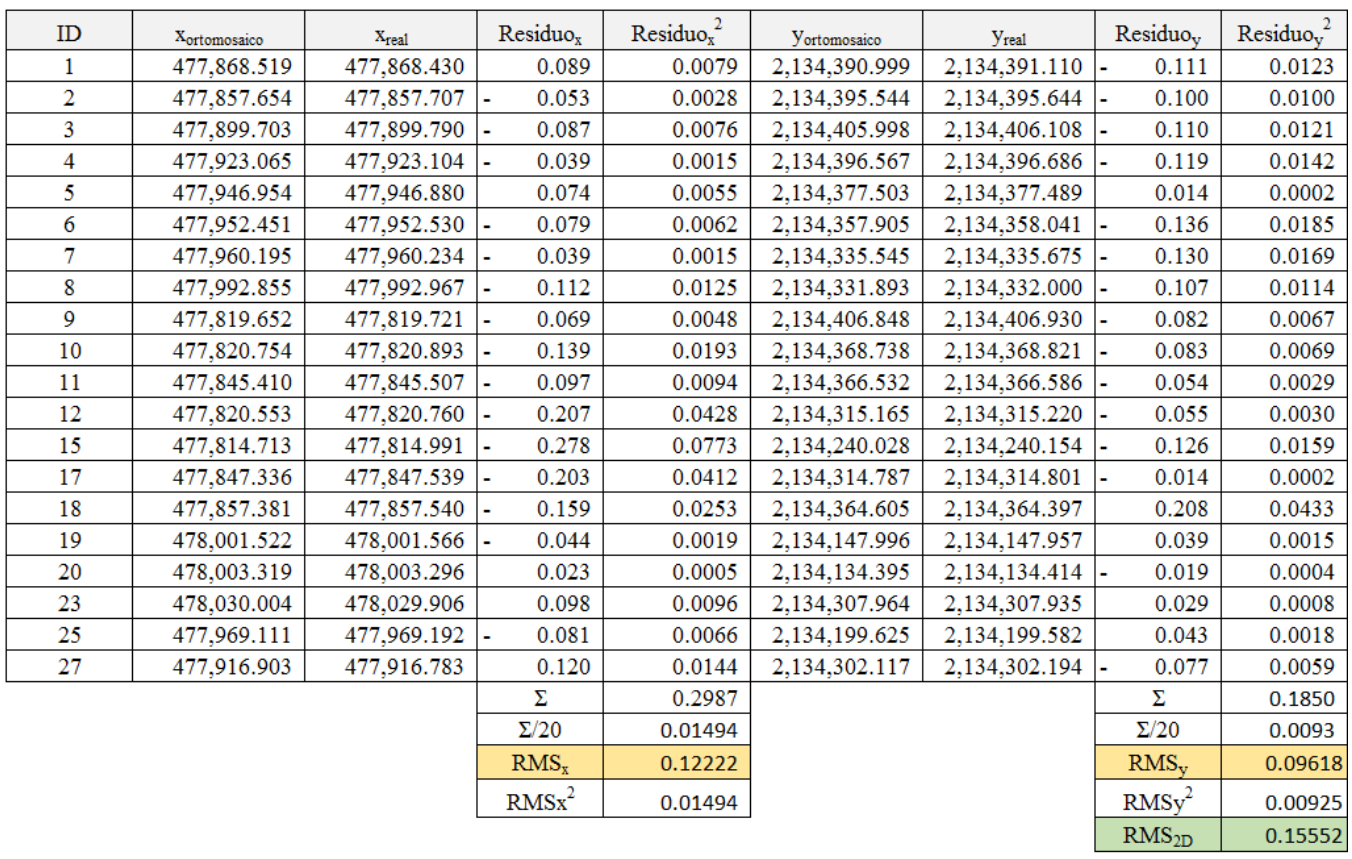

Table 4. Calculation of the RMS2D with 20 ICPs

\section{Conclusions}

- The Photogrammetric Process using UAVs is a new perspective of the Digital Photogrammetry. Although the drones have arrived to modify the way of obtaining photographs, their processing continues methodologies established since the era of Analog Photogrammetry.

- The design of the Protocol was essential for the good result of the Photo-grammetric Survey, being seen as a check-list of everything that had to be done before, during and after the flights.

- It is possible that the proposal of the Protocol (from the specific point of view of buildings surveys) may become a feasible tool to be used for surveys with 2D or 3D cadastral purposes, with the generation of orthomosaics for the update of the two-dimensional, and the generation of basic 3D models for the latter.

- The obtained Photogrammetric Products have a good spatial resolution, based on the height of the flights and the size of their GSD. 


\section{References}

1. Luhmann T., Robson S., Kyke S., Boehm J.; Close-Range Photogrammetry and 3D Imaging. De Gruyter, Germany (2014).

2. 2. Jazayeri I., Rajabifard A., Kalantari M.; A geometric and semantic evaluation of $3 D$ data sourcing methods for land and property information. Land Use Policy 36. June 2014. 219-230.

3. Caire Lomelì J.; Fotogrametría I: Fotogrametría Terrestre. Editorial Rodrìguez, Mexico City (1977).

4. Wolf P.R., Dewitt B.A., Wilkinson B.E.; Elements of Photogrammetry with Ap-plications in GIS. McGraw-Hill Education, USA. (2014).

5. Schenck T.; Introduction to Photogrammetry. Manual of the Department of Civil and Environmental Engineering and Geodetic Sciences. Ohio State University. Fall Quarter 2005. USA

6. Remondino F., El-Hakim S.; Image-based 3D Modelling: A Review. The Photo-grammetric Record Vol. 21. Number 115. September 2006. 269-291

7. Reyes Salas V. M.; Valores Arquitectónicos de la UPN Ajusco: Su Apropiación por la Comunidad Académica. Doctoral thesis. Colegio de Estudios de Posgrado de la Ciudad de México, Ixtlahuaca, Estado de México, 2013.

8. Calvo K.; So You Want to Create Maps Using Drones?. Blurb, USA (2015).

9. Cruz Reyes I.C.; Evaluación de Modelos Digitales de Elevación en el Proceso de Ortorrectificación de Imágenes Satelitales de Muy Alta Resolución. School of En-gineering UNAM, Mexico City (2015).

10. Lencinas J.D., Díaz G.M.; Incidencia de los modelos digitales de elevación en la corrección geométrica de datos satelitales QuickBird: caso del SRTM-C, SRTM-X y ASTER GDEM. Geomatics Lab. Centro de Investigación y Extensión Forestal Andino Patagónico. Argentina (2011). 